Proceedings of the 2011 Winter Simulation Conference

S. Jain, R. R. Creasey, J. Himmelspach, K. P. White, and M. Fu, eds.

\title{
VALUATION OF COLLATERALIZED DEBT OBLIGATIONS IN A MULTIVARIATE SUBORDINATOR MODEL
}

\author{
Yunpeng Sun \\ Northwestern University \\ 2145 Sheridan Road C210 \\ Evanston, IL 60208, USA
}

\author{
Rafael Mendoza-Arriaga \\ University of Texas at Austin \\ CBA 5.202, B6500, 1 University Station \\ Austin, TX 78712, USA
}

\author{
Vadim Linetsky \\ Northwestern University \\ 2145 Sheridan Road C210 \\ Evanston, IL 60208, USA
}

\begin{abstract}
The paper develops valuation of multi-name credit derivatives, such as collateralized debt obligations (CDOs), based on a novel multivariate subordinator model of dependent default (failure) times. The model can account for high degree of dependence among defaults of multiple firms in a credit portfolio and, in particular, exhibits positive probabilities of simultaneous defaults of multiple firms. The paper proposes an efficient simulation algorithm for fast and accurate valuation of CDOs with large number of firms.
\end{abstract}

\section{INTRODUCTION}

A collateralized debt obligations (CDO) is a financial claim to the cash flows generated by a portfolio of debt instruments. Cash CDOs are based on portfolios of corporate bonds, sovereign bonds, loans, mortgages, etc. $C D O$ tranches are notes of varying seniority issued against the underlying credit portfolio and deliver cash flows to investors based on the cash flows from the assets in the portfolio. Synthetic CDOs are based on portfolios of credit default swaps (CDS). A CDS is essentially an insurance contract in which a buyer of credit protection makes fixed periodic payments until contract expiration, such as five years. If there is a default on the underlying reference bond during that period, then the buyer of protection has the right to give the defaulted bond to the protection seller and receive the full face value of the bond in exchange. In effect, the value of the CDS payoff in the event of default is equal to the loss given default (LGD) on the reference bond.

Important examples of synthetic CDO contracts (also called tranche swaps) are CDOs based on the most liquid U.S. corporate credit derivative index, the Dow Jones CDX North America Investment Grade Index. It is based on a basket of CDS contracts on 125 U.S. firms with investment grade debt. The CDX index itself trades just like a single-name CDS (index default swap), with a defined premium based on the equally weighted basket of its 125 constituents. Index CDO tranches of varying seniority are issued tied to the CDX index. The iTraxx index is a similar index of 125 European investment grade firms. While CDOs based on major indexes such as CDX and iTraxx are the most liquid CDOs, a CDO can be based on any credit sensitive portfolio. A wide variety of customized (bespoke) CDOs have been issued prior to the global credit crisis of 2007-2009, some of them on highly risky portfolios, such as subprime mortgages. The key to CDO valuation is the understanding and modeling of dependence among default events in the credit portfolio. Underestimating default dependence and, hence, the probability of multiple 


\section{Sun, Mendoza-Arriaga, and Linetsky}

defaults occurring in a short period of time (default clustering) would result in major underestimation of risk and, hence, major mispricing of senior tranches. This is what happened in years preceding the credit crisis, when financial institutions, credit rating agencies and investors often used simplified models with weak default dependence that implied very low probabilities of multiple defaults that resulted in significant misestimation of risk and misvaluation of CDOs. As is now well known, some senior CDO tranches rated AAA by credit rating agencies (the highest rating for safety and low risk of a credit instrument) suffered major losses during the credit crisis.

CDO valuation models generally fall into one of two categories - bottom-up or top-down. In a bottom-up approach, one models default dynamics of each individual obligor in the credit portfolio, and then aggregates the component losses to construct the portfolio loss process. The dependence structure of defaults across different obligors is modeled in a number of ways. Copula-based models (Embrechts (2009) gives an excellent introduction to the copula approach.) couple marginal distributions via preselected joint distributions. For example, Li (2008) proposes Gaussian copula, where the preselected joint distribution is multidimensional Normal distribution. The model was once considered industry standard, but was shown to significantly underestimate portfolio risk, because it fails to generate correlated large losses and default clustering in particular. By constructions, these models are also static in a sense that they do not model the credit dynamics of each obligor. In a different approach, intensity-based bottom-up models describe dependence structure by modeling stochastic and correlated default intensities (stochastic default arrival rates) of obligors in the portfolio. The default time of one firm is modeled as the first jump time of a doubly stochastic Poisson process with stochastic arrival rate. The single name intensity-based models are introduced by Jarrow and Turnbull (1995), Lando (1998), and Duffie and Singleton (1999), and stemming from them, the multi-name intensity-based model was originally proposed by Duffie and Gârleanu (2001) and extended by Mortensen (2006) and many others. Generally speaking, the bottom-up approach based on correlated stochastic default intensities offers a consistent dynamic framework to model both single-name as well as portfolio dynamics, which makes hedging and managing risk consistent. The drawback is that intensity based models have difficulties producing high levels of default correlation and, in particular, the probability of simultaneous defaults of multiple obligors vanishes. This is intuitively clear since even if the default intensities of two firms simultaneously experience significant upward jumps, their integrals (hazards) will take some time to "feel" that increase in the integrand and, hence, the probability of simultaneous default of two obligors in the correlated intensity model vanishes. Another drawback is computational, requiring significant computational resources to simulate each individual obligor's dynamics and aggregate to the portfolio level.

In contrast, top-down approaches directly model the portfolio loss process. Representative works are Errais, Giesecke, and Goldberg (2007), Cont and Minca (2007), Giesecke and Kim (2007), and Longstaff and Rajan (2008), among others. The top-down approach models portfolio loss dynamics rather than individual losses. As a result, the size of the portfolio have little influence on the computational effort, as the dimension of the problem is effectively reduced to several factors included in the model of a one-dimensional portfolio loss process. The drawback is that the consistency with single-name dynamics is generally lost. That creates difficulties with hedging multi-name and single-name credit derivatives consistently.

During the credit crisis, multiple defaults occurred in the fall of 2008 in the very short period of time. It is interesting to point out that, just prior to the credit crisis, a number of researchers, Jorion and Zhang (2007), Das, Duffie, Kapadia, and Saita (2007) and Longstaff and Rajan (2008) among others, have provided empirical evidence of default clustering phenomena and pointed out that the existing models used in the industry at the time failed to take it into account and, more generally. The credit crisis reinforced the need to incorporate strong enough default dependence and the possibility of default clustering in credit modeling. From the bottom-up perspective, original intensity-based models are incapable to capture some excess correlation that exists due to default clustering, as shown in Das, Duffie, Kapadia, and Saita (2007). To incorporate richer dependency, Zheng (2009) propose to change default intensities of firms if one firm 
defaults. Peng and Kou (2009), Mai and Scherer (2009) and Mendoza-Arriaga and Linetsky (2010) extend the intensity-based models and incorporate default clustering by modeling the hazard process directly as a jump process, rather than modeling the intensity. When the hazard processes of multiple obligors jump above their thresholds at the same time under the influence of a common factor experiencing a shock, simultaneous defaults of multiple obligors occur in such models.

In the present paper we apply the model proposed by Sun, Mendoza-Arriaga, and Linetsky (2010), where the authors use multidimensional Lévy subordinators to provide an alternative construction of the celebrated Marshall-Olkin (MO) multivariate exponential distribution proposed by Marshall and Olkin (1967) as a model for failure times with the possibility of simultaneous failure (default). The Marshall-Olkin class of distributions has been studied in reliability literature, and it has a number of attractive features to model dependent lifetimes of components in a system, including the possibility of simultaneous failures. But in order to sample from an $n$-dimensional MO distribution, according to the original MO fatal shock construction, one needs to simulate $2^{n}$ independent exponential random variables. That drastically limits the applicability to small dimensional problems only. In a typical credit portfolio, the number of obligors could be from hundreds to thousands, thus making general MO distributions infeasible to simulate. Sun, Mendoza-Arriaga, and Linetsky (2010) prove that every $n$-dimensional MO distribution whose parameters satisfy a certain condition can be constructed as the distribution of the first passage times of coordinates of an $n$-dimensional Lévy subordinator process above $n$ independent unit mean exponential random variables. This new construction of the class of MO distributions breaks the curse of dimensionality and facilitates efficient simulation.

The present paper applies the results of Sun, Mendoza-Arriaga, and Linetsky (2010) to the modeling and valuation of CDOs. The default time of each obligor is modeled as the first passage times of the corresponding coordinate of a multidimensional subordinator above a unit mean exponential random variables. Each coordinate of the multidimensional subordinator serves as the hazard process of the corresponding obligor. Each coordinate of the multidimensional subordinator is a linear combination of several independent one-dimensional subordinators, with which we represent different risk factors. The model shows explicit and rich dependence structure that, we believe, is large enough in any credit risk application. In this framework, simultaneous defaults happen when different coordinates of the multivariate subordinator jump and pass their respective thresholds at the same time due to a jump in a common factor. The marginal and joint survival probabilities can be easily expressed in closed form in terms of the Laplace exponent of the Lévy subordinator. Nevertheless, for large portfolios like CDX and iTraxx, the explosion of combinatorial terms in the sums entering the expressions makes it infeasible to value CDO tranches analytically. In contrast, the efficient simulation in this model is straightforward, with the computational effort growing linearly in the number of obligors in the CDO's reference portfolio.

The rest of the paper is organized as follows. In section 2, we survey multi-dimensional subordinator processes and, in particular, discuss the linear factor model - a class of $n$-dimensional subordinators that can be written as linear combinations of independent one-dimensional subordinators. In section 3, we introduce our model for dependent defaults based on the linear factor model and discuss its properties. Section 4 introduces CDO contracts, gives the CDO tranche pricing formulas, and shows the efficient simulation algorithm. In section 5, we provide a simulation experiment, where we simulate CDO tranche spreads derived on a group of 125 obligors.

\section{MULTIVARIATE SUBORDINATORS}

An $n$-dimensional subordinator is a Lévy process in $\mathbb{R}_{+}^{n}=[0, \infty)^{n}$ starting at the origin that is nondecreasing in each of its coordinates. That is, each of its coordinates is a one-dimensional subordinator, a one-dimensional non-decreasing Lévy process starting at zero. Recall that a Lévy process is a process with right-continuous with left limits sample paths, with stationary and independent increments, and continuous in probability. The ( $n$-dimensional) Laplace transform of an $n$-dimensional subordinator is given by the 
Lévy-Khintchine formula (here $u_{i} \geq 0$ and $\langle\mathbf{u}, \mathbf{v}\rangle=\sum_{i=1}^{n} u_{i} v_{i}$ ):

$$
\mathbb{E}\left[e^{-\left\langle\mathbf{u}, T_{t}\right\rangle}\right]=e^{-t \phi(\mathbf{u})}
$$

with the Laplace exponent given by:

$$
\phi(\mathbf{u})=\langle\gamma, \mathbf{u}\rangle+\int_{\mathbb{R}_{+}^{n}}\left(1-e^{-\langle\mathbf{u}, \mathbf{s}\rangle}\right) v(d \mathbf{s}),
$$

where $\gamma \in \mathbb{R}_{+}^{n}$ is the drift of the subordinator and the Lévy measure $v$ is a $\sigma$-finite measure on $\mathbb{R}^{n}$ concentrated on $\mathbb{R}_{+}^{n} \backslash\{0\}$ such that $\int_{\mathbb{R}_{+}^{n}}(\|\mathbf{s}\| \wedge 1) v(d \mathbf{s})<\infty$. Thus, the multivariate subordinator is a process with positive jumps in each of its coordinates governed by the Lévy measure $v$ and with non-negative drift $\gamma$. The integrability condition means that the Lévy measure is integrable away from the origin, while the singularity at the origin is such that $\|\mathbf{s}\| v(d \mathbf{s})$ is integrable.

A class of multi-dimensional subordinators convenient for applications can be constructed as follows. Let $S_{t}^{a}$ be $m$ independent one-dimensional subordinators and $A$ an $n \times m$ matrix with non-negative entries $A_{i, a} \geq 0$. Define

$$
T_{t}^{i}=\sum_{a=1}^{m} A_{i, a} S_{t}^{a}, \quad i=1, \ldots, n .
$$

Then the $\mathbb{R}_{+}^{n}$-valued process $T_{t}$ is an $n$-dimensional subordinator. In this structure, each independent subordinator $S^{a}$ contributes to each coordinate $T^{i}$ of the $n$-dimensional subordinator $T$. The coefficient $A_{i, a}$ is the corresponding loading coefficient. We call this class of multi-dimensional subordinators the linear factor model. The Laplace exponent is given by:

$$
\phi(u)=\sum_{a=1}^{m} \phi_{a}\left(v_{a}\right) \text { with } v_{a}=\sum_{i=1}^{n} A_{i, a} u_{i}
$$

where $\phi_{a}(v)$ are the Laplace exponents of the $m$ independent one-dimensional subordinators $S^{a}$. The drift vector and Lévy measure of $T$ are:

$$
\gamma_{i}=\sum_{a=1}^{m} A_{i, a} \gamma_{a}, v(G)=\sum_{a=1}^{m} v_{a}\left(G_{a}\right), G \in \mathscr{B}\left(\mathbb{R}_{+}^{n}\right)
$$

where $\gamma_{a}$ and $v_{a}$ are the drift and Lévy measure of $S^{a}$, and $G_{a}=\left\{s_{a} \geq 0: A\left(0, \ldots, s_{a}, \ldots, 0\right)^{\top} \in G\right\}$, where $s_{a}$ is in the $a$ th place. The matrix $A$ defines the covariance:

$$
\operatorname{Cov}\left(T_{t}^{i}, T_{t}^{j}\right)=t \Sigma_{i j}, \Sigma_{i j}=-\sum_{a=1}^{m} \phi_{a}^{\prime \prime}(0) A_{i, a} A_{j, a},
$$

where $-\phi_{a}^{\prime \prime}(0) t$ is the variance of the $a$ th independent subordinator $S_{t}^{a}$. Further examples of multi-dimensional subordinators can be found in Barndorff-Nielsen and Sato (2001).

A family of one-dimensional subordinators important in applications is defined by the following three-parameter family of Lévy measures:

$$
v(d s)=C s^{-\alpha-1} e^{-\eta s} d s
$$

with $C>0, \eta \geq 0$, and $\alpha<1$. For $\alpha \in(0,1)$ these are the so-called tempered stable subordinators (exponentially dampened versions of the stable subordinators with $v(d s)=C s^{-\alpha-1} d s$ ). The special case with $\alpha=1 / 2$ is the inverse Gaussian (IG) subordinator (see Barndorff-Nielsen (1998)). The limiting case $\alpha=0$ is the gamma subordinator (see Madan and Seneta (1990) and Madan, Carr, and Chang (1998)). The 


\section{Sun, Mendoza-Arriaga, and Linetsky}

processes with $\alpha \in[0,1)$ are infinite activity processes. The processes with $\alpha<0$ are compound Poisson processes with gamma distributed jump sizes (in particular, with exponential jumps when $\alpha=-1$ ). The Laplace exponent is given by:

$$
\phi(\lambda)=\left\{\begin{array}{cc}
-C \Gamma(-\alpha)\left((\lambda+\eta)^{\alpha}-\eta^{\alpha}\right), & \alpha \neq 0 \\
C \ln (1+\lambda / \eta), & \alpha=0
\end{array},\right.
$$

where $\Gamma(x)$ is the gamma function. The parameter $C$ affects the intensity of jumps of all sizes simultaneously. The parameter $\eta$ controls the decay rate of large jumps. The parameter $\alpha$ controls the relative importance of small jumps in sample paths of the subordinator. Further information on subordinators can be found in Bertoin (1996)), Sato (1999) and Schilling, Song, and Vondracek (2010). For applications in finance see Boyarchenko (2002), Cont and Tankov (2004) and Schoutens (2003).

In general, the transition function of the subordinator with a given Laplace exponent has to be recovered numerically by inverting the Laplace transform. In special cases of gamma, inverse Gaussian, and compound Poisson subordinators with gamma distributed jumps the transition functions are known in closed form. In particular, the state $S_{t}$ of the gamma subordinator at time $t>0$ has the gamma density:

$$
f_{S_{t}}^{\text {Gamma }}(s)=\frac{s^{k-1}}{\theta^{k} \Gamma(k)} \exp \left(-\frac{s}{\theta}\right), \quad s>0, \quad k=C t, \quad \theta=\frac{1}{\eta} .
$$

The state of the IG subordinator at time $t>0$ has the IG density:

$$
f_{S_{t}}^{I G}(s)=\sqrt{\frac{\lambda}{2 \pi s^{3}}} \exp \left(-\frac{\lambda(s-\mu)^{2}}{2 \mu^{2} s}\right), \quad s>0, \quad \lambda=2 \pi C^{2} t^{2}, \quad \mu=\sqrt{\frac{\pi C^{2} t^{2}}{\eta}} .
$$

The linear factor model (2) defines a special class of multi-dimensional subordinators convenient for simulation. In this class the dependence structure is explicitly described by the matrix $A$ that decomposes the $n$-dimensional subordinator into $m$ independent factors $S$. If the independent factors are subordinators with known transition densities, then the simulation of the $n$-dimensional subordinator is reduced to the simulation of $m$ independent one-dimensional subordinators with known densities. For general subordinators, their dependence structure can be described by the Lévy copula introduced by Kallsen (1986).

\section{THE MULTIVARIATE SUBORDINATOR MODEL OF DEPENDENT DEFAULT TIMES}

\subsection{Model Set-Up}

Consider a credit portfolio of $n$ firms. We use the linear factor model (2) to describe dependence structure among defaults of the $n$ firms as follows. Let $S_{t}^{c}, c=1, \ldots, m$ be $m$ independent one-dimensional subordinators representing common factors affecting multiple firms in the portfolio. Let $S_{t}^{i}, i=1, \ldots, n$, be $n$ independent one-dimensional subordinators representing idiosyncratic (firm-specific) factors affecting only the $i$ th firm. Define an $n$-dimensional subordinator as follows:

$$
T_{t}^{i}=\sum_{c=1}^{m} A_{i, c} S_{t}^{c}+S_{t}^{i}
$$

where $A_{i, c} \geq 0, c=1, \ldots, m$ are the factor loadings of the common factors. Define the time of default of the $i$ th firm as follows:

$$
\tau_{i}=\inf \left\{t \geq 0: T_{t}^{i}+f_{i}(t) \geq E_{i}\right\}
$$

where $T_{t}^{i}$ is the $i$-th coordinate of the previously defined $n$-dimensional subordinator $T, E_{i}$ are i.i.d exponential unit mean random variables and independent of $S_{t}^{c}$ and $S_{t}^{i}$, and $f_{i}(t), i=1, \ldots, n$ are deterministic functions of time needed to calibrate to any given single-name term structure of CDS spreads for each individual firm. 


\subsection{Model Properties}

The default dependence structure among obligors is explicit and rich in this model. As shown in (4), the correlation structure is captured by $A_{i, c}$ and $S_{t}^{c}$. By choosing the loading coefficients, one controls the influence of the common factors $S_{t}^{c}$ on different firms in the portfolio. The common factors may include systematic (economy-wide), industry-specific or geographic-specific factors, which can all be incorporated as multiple subordinators. Different types of subordinators may be used to model specific feature. For instance, subordinators with large but less frequent jumps are suitable to model systematic factors that proxy for major market shocks affecting everyone, while those with small and frequent jumps are suitable to model firm-specific factors.

As proved in Sun, Mendoza-Arriaga, and Linetsky (2010), when $f_{i}=0$, the distribution of the random vector $\left(\tau_{i}\right)$ of default times is a Marshall-Olkin multivariate exponential distribution with parameters expressed in terms of the Laplace exponents of subordinators and loading coefficient $A$. As such, the distribution has singular components that model simultaneous defaults of multiple obligors. In particular, the probability of simultaneous default of two obligors $i$ and $j$ is given explicitly by (in the general case with possibly non-zero $f_{i}$ and $f_{j}$ ):

$$
\mathbb{P}\left(\tau_{i}=\tau_{j}\right)=\frac{\varphi_{i}+\varphi_{j}-\varphi_{i, j}}{\varphi_{i, j}} e^{-f_{i}(t)-f_{j}(t)},
$$

where $\varphi_{k}=\phi_{k}(1)+\sum_{c=1}^{m} \phi_{c}\left(A_{k, c}\right), k=i, j$ and $\varphi_{i, j}=\phi_{i}(1)+\phi_{j}(1)+\sum_{c=1}^{m} \phi_{c}\left(A_{i, c}+A_{j, c}\right)$.

The model can match arbitrary marginal survival probabilities of every obligor, thus facilitating explicit calibration to single-name CDS data. The joint survival probabilities can then be easily calculated in closed form. Let $\phi_{c}(\cdot)$ and $\phi_{i}(\cdot)$ be the Laplace exponents of $S_{t}^{c}$ and $S_{t}^{i}$. The marginal survival probability $q_{i}(t)$ is given by

$$
q_{i}(t)=\mathbb{P}\left(\tau_{i}>t\right)=\mathbb{E}\left[\mathbb{P}\left(E_{i}>T_{i}+f_{i}(t) \mid T_{i}\right)\right]=\mathbb{E}\left[e^{-T_{i}-f_{i}(t)}\right]=e^{-t \varphi_{i}-f_{i}(t)},
$$

where $\varphi_{i}$ is defined above. Note that without $f_{i}(t)$, the single name default time follows exponential distribution. By adding $f_{i}(t)$, we can match arbitrary marginal default time distributions. Given a set of common and firm-specific subordinators, $f_{i}(t), i=1, \ldots, n$ are chosen equal to $-\ln q_{i}(t)-t \varphi_{i}$ to match single-name default probabilities. Let $\Theta$ be some subset of $K$ obligors in the portfolio with indexes $i_{k}, k=1, \ldots, K$. Then the joint survival probability for this subset of obligors is:

$$
\mathbb{P}\left(\tau_{i_{1}}>t, \ldots, \tau_{i_{K}}>t\right)=\mathbb{E}\left[e^{-\sum_{k=1}^{K}\left(T_{t}^{i_{k}}+f_{i_{k}}(t)\right)}\right]=e^{-\sum_{k=1}^{K} \phi_{i_{k}}(1) t-\sum_{c=1}^{m} \phi_{c}\left(\sum_{k=1}^{K} A_{i_{k}, c}\right) t-\sum_{k=1}^{K} f_{i_{k}}(t)} .
$$

Another useful feature of this model specification is conditional default independence conditional on common factors $S_{t}^{c}$. This allows for significant computational efficiency in CDO valuation. Instead of explicitly specifying idiosyncratic subordinators $S_{t}^{i}$, as well as $f_{i}(t)$, we can directly take single-name survival probabilities as model inputs. This is similar to the approach taken in Peng and Kou (2009) in a different model specification with common and idiosyncratic factors. On one hand, single-name and multi-name credit instruments can be modeled in one framework, hence facilitating hedging and risk management. On the other, marginal (risk-neutral) survival probabilities can be easily extracted from market quotes of single name CDS. More precisely, given common factors $S_{t}^{c}, c=1, \ldots, m$, firms will default independently, and the conditional survival probability is given by:

$$
q_{i}^{c}(t)=\mathbb{P}\left(\tau_{i}>t \mid S_{t}^{c}\right)=\mathbb{E}\left[e^{-S_{t}^{i}-\sum_{c=1}^{m} A_{i, c} S_{t}^{c}-f_{i}(t)} \mid S_{t}^{c}\right]=e^{-t \phi_{i}(1)-\sum_{c=1}^{m} A_{i, c} S_{t}^{c}-f_{i}(t)} .
$$

Comparing (12) to (10), we immediately derive the relationship of the conditional survival probability and the unconditional survival probability. That is,

$$
q_{i}^{c}(t)=q_{i}(t) e^{-\sum_{c=1}^{m}\left(A_{i, c} S_{t}^{c}-\phi_{c}\left(A_{i, c}\right)\right)} .
$$


This is particularly useful in Monte Carlo simulation, because we can independently simulate the status of firms.

\subsection{Application to Modeling Credit Portfolios}

Define the default indicator process $D_{t}^{i}=\mathbf{1}_{\left\{\tau_{i} \leq t\right\}}$, which indicates if the company $i$ has defaulted or not by time $t$. Then the cumulative portfolio loss process $L_{t}$ is given by:

$$
L_{t}=\sum_{i=1}^{n} w_{i} L G D_{i} D_{t}^{i}
$$

where $L G D_{i}$ is the loss given default on the $i$ th obligor's debt, and $w_{i}$ is the corresponding portfolio weight (total exposure to the $i$ th obligor). To simplify notion and without loss of generality, throughout this paper we assume that the total portfolio notional amount is $N=1$.

CDO tranches can be viewed as contingent claims on the loss process $L_{t}$ and, hence, on default indicators. In principle, the expectation of any function of $D_{t}^{i}$ can be written down in closed form in terms of the summation taken over $2^{n}$ possible realizations of the binary random variables $D_{t}^{i}$ with probabilities calculated by using joint survival probabilities (11). As portfolio size increases, this method quickly becomes infeasible. In contrast, our model specification provides a direct way to simulate the loss process.

First, we specify the one-dimensional subordinators as those with closed form transition probability functions, such as gamma, inverse Gaussian, and compound Poisson with gamma distributed jumps, so that they can be easily simulated at any given time $t$. We then simulate unit mean exponential random variables and compare them to the subordinator coordinates plus $f_{i}(t)$, which lead to the knowledge of default indicators $D_{t}^{i}$.

An additional efficiency arises from the observation that one does not actually need to specify firmspecific subordinators $S_{t}^{i}$ and functions $f_{i}(t), i=1, \ldots, n$. Rather, single name marginal survival probabilities can be used as the direct model input. The common factors $S_{t}^{c}, c=1, \ldots, m$ are simulated first. Conditional on that, derive the conditional survival probabilities through (13) for all $i$. The indicator $D_{t}^{i}$ follows the Bernoulli $\left(1-q_{i}^{c}(t)\right)$ distribution. Thus, single-name survival probabilities are used as direct model input, resulting in consistency of the multi-name valuation model with single-name CDS. Instead of simulating $n$ firm specific subordinators and $n$ exponential random variables, we generate $n$ Bernoulli random variables. In the next section, we will discuss the details of this simulation algorithm for CDO valuation.

\section{PRICING COLLATERALIZED DEBT OBLIGATIONS}

\subsection{Introduction to CDO Markets}

In this paper we focused on synthetic CDOs (also called tranche swaps) on credit indexes. To give an example, we describe index tranche swaps tied to the most liquid U.S. corporate credit derivatives index, the Dow Jones CDX North American Investment Grade Index (CDX.NA.IG). It is based on a basket of CDS contracts on 125 U.S. firms with investment grade debt. The CDX index itself trades just like a single-name CDS contract (index default swap), with a defined premium based on the equally weighted basket of its 125 constituents. Every six months in March and September, the index is revised and reissued with some downgraded and illiquid firms being dropped from the index and some new ones added. The first CDX.NA.IG index was issued in 2003, and since then the indexes are numbered sequentially. The CDO tranches are divided into intervals $0 \%-3 \%, 3 \%-7 \%, 7 \%-15 \%, 15 \%-100 \%$ of the total losses on the index portfolio. The most junior tranche experiences no losses if the total losses of the underlying portfolio are zero, and experiences the loss of $100 \%$ of the tranche notional amount if the total losses on the underlying portfolio equal or exceed $3 \%$ of the portfolio principal. If the underlying portfolio losses are between zero and $3 \%$, the corresponding junior tranche losses are linearly interpolated between zero and one. The second most junior tranche (junior mezzanine) with the attachment level of 3\% and the detachment level of $7 \%$ experiences no loss if the underlying portfolio losses do not exceed $3 \%$ of portfolio principal (as those 
losses are absorbed by the equity tranche). The losses are 1.00 or $100 \%$ if the total losses on the underlying portfolio equal or exceed $7 \%$ of the portfolio principal. The most senior tranche with the attachment level of $15 \%$ and the detachment level of $100 \%$ experiences no loss if underlying portfolio losses do not exceed $15 \%$. For underlying portfolio losses between $15 \%$ and $100 \%$, the corresponding tranche losses are linearly interpolated between zero and one.

The iTraxx index is a similar index for 125 European investment grade firms. iTraxx CDOs are structured similarly to CDX CDOs, but have different attachment points. CDX and iTraxx credit index families also include other indexes, such as CDX High Yield North America and similar iTraxx indexes based on European lower rated obligors. Although CDOs based on major indexes are the most liquid synthetic CDOs, a synthetic tranche can be based on any portfolio.

\subsection{CDO Tranche Spreads Valuation Formula}

The mathematical formulation of the CDO tranche pricing problem is as follows. Let the interval $[0,1]$ be partitioned into $J$ contiguous tranches $\left[\varepsilon_{j-1}, \varepsilon_{j}\right), j=1, \ldots, J$, with $\varepsilon_{0}=0$ and $\varepsilon_{J}=1$ (corresponding to $100 \%)$. So for the $j$ th tranche, $\varepsilon_{j-1}$ and $\varepsilon_{j}$ are the attachment and detachment points respectively. The normalized cumulative loss up to time $t$ of the tranche $j$ is defined as:

$$
L_{t}^{\left[\varepsilon_{j-1}, \varepsilon_{j}\right)}=\frac{\left(L_{t}-\varepsilon_{j-1}\right)^{+}-\left(L_{t}-\varepsilon_{j}\right)^{+}}{\varepsilon_{j}-\varepsilon_{j-1}},
$$

where $L_{t}$ is the cumulative portfolio loss process defined in Eq.(14). It is normalized so that the loss is equal to one (100\% of the principal) when $L_{t} \geq \varepsilon_{j}$.

A CDO contract specifies the maturity $T$ and a set of payment dates $0<t_{1}<t_{2}<\ldots<t_{K}=T$. Typically, the payment is made quarterly. At each $t_{k}, k=1, \ldots, K$, the protection seller receives a fixed premium payment from the protection buyer at the rate of $S^{\left[\varepsilon_{j-1}, \varepsilon_{j}\right)}$ per annum on the remaining notional $1-L_{t_{k}}^{\left[\varepsilon_{j-1}, \varepsilon_{j}\right)}$ of the tranche $j$. In exchange for this premium payment, the protection buyer (CDO issuer) is covered for the loss incurred between time $t_{k-1}$ and $t_{k}$. Therefore, at time $t_{0}=0$, the present value of the premium leg of the tranche $j$ is given by

$$
\mathbb{E}\left[\sum_{k=1}^{K} D F\left(0, t_{k}\right) S^{\left[\varepsilon_{j-1}, \varepsilon_{j}\right)}\left(t_{k}-t_{k-1}\right)\left(1-L_{t_{k}}^{\left[\varepsilon_{j-1}, \varepsilon_{j}\right)}\right)\right],
$$

where $D F(0, t)$ is the risk free discount factor from $t$ to time 0 . The present value of the protection leg of the tranche $j$ is:

$$
\mathbb{E}\left[\sum_{k=1}^{K} D F\left(0, t_{k}\right)\left(L_{t_{k}}^{\left[\varepsilon_{j-1}, \varepsilon_{j}\right)}-L_{t_{k-1}}^{\left[\varepsilon_{j-1}, \varepsilon_{j}\right)}\right)\right] .
$$

The spread of tranche $j$ is obtained by setting the present value of the premium leg equal to the present value of the protection leg:

$$
S^{\left[\varepsilon_{j-1}, \varepsilon_{j}\right)}=\frac{\mathbb{E}\left[\sum_{k=1}^{K} D F\left(0, t_{k}\right)\left(L_{t_{k}}^{\left[\varepsilon_{j-1}, \varepsilon_{j}\right)}-L_{t_{k-1}}^{\left[\varepsilon_{j-1}, \varepsilon_{j}\right)}\right)\right]}{\mathbb{E}\left[\sum_{k=1}^{K} D F\left(0, t_{k}\right)\left(t_{k}-t_{k-1}\right)\left(1-L_{t_{k}}^{\left[\varepsilon_{j-1}, \varepsilon_{j}\right)}\right)\right]} .
$$

In some CDO contracts the premium is paid via an upfront fee payment, $U^{\left[\varepsilon_{j-1}, \varepsilon_{j}\right)}$, as well as the running annual spread $S^{\left[\varepsilon_{j-1}, \varepsilon_{j}\right)}$. In that case, at each payment date, the protection buyer pays a fixed running spread $S^{\left[\varepsilon_{j-1}, \varepsilon_{j}\right)}$ that is fixed in the contract, such as 100 basis points per year, and a quoted upfront fee. In that 
case, the upfront fee is equal to:

$$
\begin{aligned}
U^{\left[\varepsilon_{j-1}, \varepsilon_{j}\right)}= & \mathbb{E}\left[\sum_{k=1}^{K} D F\left(0, t_{k}\right)\left(L_{t_{k}}^{\left[\varepsilon_{j-1}, \varepsilon_{j}\right)}-L_{t_{k-1}}^{\left[\varepsilon_{j-1}, \varepsilon_{j}\right)}\right)\right] \\
& -S^{\left[\varepsilon_{j-1}, \varepsilon_{j}\right)} \mathbb{E}\left[\sum_{k=1}^{K} D F\left(0, t_{k}\right)\left(t_{k}-t_{k-1}\right)\left(1-L_{t_{k}}^{\left[\varepsilon_{j-1}, \varepsilon_{j}\right)}\right)\right] .
\end{aligned}
$$

CDO tranche swaps based on different indexes have different quoting conventions. For example, in CDOs based on CDX.NA.IG, starting with Series 15, the equity tranche 0-3\% is traded with upfront fee plus 500bps, 3-7 and 7-10 tranche will trade on a running coupon of 100bps + upfront payment and 15-100 tranche trades fee plus a running spread of $25 \mathrm{bps}$.

\subsection{CDO Valuation by Monte Carlo Simulation}

The practical difficulty with evaluating the valuation formulas (18) and (19) analytically is the curse of dimensionality in evaluating the expectations. As an alternative, we simulate the underlying subordinator model and the resulting portfolio loss process. In the linear factor subordinator model (7), $S_{t}^{c}, c=1, \ldots, m$ and $S_{t}^{i}, i=1, \ldots, n$ are independent one-dimensional subordinators, where we assume that each subordinator has an explicitly known transition function (cf. Eqs.(5-6) for the gamma or inverse Gaussian subordinators). We also exploit the property of conditional independence of defaults given the common factors, as discussed previously. For the valuation of CDOs, we need to simulate default indicators $D_{t}^{i}$ on a grid of payment dates $0<t_{1}<\ldots,<t_{K}=T$. For each replicate at $t_{k}, k=1, \ldots, K$, we simulate default indicators, and compute portfolio loss $L_{t_{k}}, k=1, \ldots, K$ as in Eq.(14). We thus have the following simulation algorithm to evaluate CDOs.

\section{Algorithm 1 (CDO Simulation)}

1. For each independent common subordinator $S_{t}^{c}, c=1, \ldots, m$, generate samples paths $S_{t_{k}}^{c}, k=1, \ldots, K$ on a discrete grid of time points corresponding to the payment dates (e.g., quarterly).

2. For each $i=1, \ldots, n$, compute the conditional survival probability $q_{i}^{c}\left(t_{k}\right), k=1, \ldots, K$ as in Eq.(13), using the unconditional survival probabilities, simulated common subordinators $S_{t_{k}}^{c}$, and the Laplace exponents of the common subordinators.

3. Generate default indicators $D_{i, k}=D_{t_{k}}^{i}$ following Bernoulli $\left(1-q_{i}^{c}\left(t_{k}\right)\right)$.

4. Compute the cumulative portfolio loss $L_{t_{k}}$ at each time $t_{k}, k=1, \ldots, K$.

5. Compute tranche spreads (18) or upfront fees (19) according to the contract specification.

Executing the simulation algorithm provides the estimate and the confidence interval of the running spread or the upfront fee for each CDO tranche. The computational effort requires to simulate a total of $n$ independent Bernoulli random variables, and $m \times K$ random variables required to simulate the common subordinators $S_{t}^{c}$ on the discrete grid of time points $t_{k}$, such as gamma or inverse Gaussian.

\section{CALIBRATION TO MARKET DATA}

In this section we show an example of consistent joint calibrating our subordinator model to market spreads of 5 year CDO based on CDX.NA.IG, as well as individual 3 year and 5 year CDS spreads of the 125 constituent companies in the index. We use the market quotes on Nov 02 2010, when Series 15 is the on-the-run index. The tranche swaps are quoted in 4 tranches. The equity tranche $0-3 \%$ is traded with a quoted upfront fee payment plus the fixed $500 \mathrm{bps}$ annual running spread, the two mezzanine tranches $3-7 \%$ and $7-10 \%$ are traded with running annual of $100 \mathrm{bps}$ plus the quoted upfront payment, and the senior $15-100 \%$ tranche is traded with an upfront fee plus a running spread of $25 \mathrm{bps}$. The portfolio is equally weighted, and to simplify analysis we assume that all obligors have the same loss-given-default 


\section{Sun, Mendoza-Arriaga, and Linetsky}

of $60 \%$. In addition, we extract discount factors from LIBOR and Swap rates curves for that day. All the market data were obtained from Bloomberg.

The default times of 125 companies $\left(\tau_{1}, \ldots, \tau_{n}\right)$ are modeled using the linear factor model as in Eq.(8). In particular, the $n$-dimensional subordinator is specified as follows,

$$
T_{t}^{i}=S_{t}^{i}+\sum_{s=1}^{2} H_{i, s} S_{t}^{s}+\sum_{g=1}^{10} G_{i, g} S_{t}^{g}, \quad i=1, \ldots, n .
$$

Here $S_{t}^{i}, i=1, \ldots, n$ are the idiosyncratic factors, while $S_{t}^{s}, s=1,2$ are two systematic factors that affect each obligor in the portfolio with the corresponding loading coefficient denoted as $H_{i, s}$. Systematic factors are proxies for the state of the global economy. Furthermore, each company belongs to one of the 10 industry sectors according Global Industry Classification Standard. Thus, we assign each industry sector a group factor $S_{t}^{g}$. $G_{i, g}$ is the corresponding loading coefficient, and it is positive only if company $i$ is in sector $g$. Group factors are independent of each other, but share the same parameter specifications to reduce the total number of parameters in the model and to simplify calibration.

The $S_{t}^{s}, s=1,2$ and $S_{t}^{g}, g=1, \ldots, 10$ are the twelve common factors $S^{c}$ within the framework of the original model (7). In this example, we select the first systematic factor to be a gamma subordinator, the second systematic factor to be a compound Poisson process with gamma distributed jumps, and 10 group factors as gamma subordinators. The idiosyncratic factors are not specified as we do not need to simulate them, as discussed in section 4 .

Let $\Omega$ denote the parameters set of all the systematic and group subordinators. Our goal is to determine $H_{i, s}, G_{i, g}$ and $\Omega$ that minimize the error between the model produced spreads and the market quotes. We apply a two-step procedure in calibration.

First, we match the individual CDS spreads of underlying companies. In this example, we assume $f_{i}(t)=0$ for all $i$, so that the marginal single-name default time distributions are exponential $-\mathbb{P}\left(\tau_{i}>\right.$ $t)=\exp \left(-t \varphi_{i}\right)$. We then choose $\varphi_{i}$ via the least squares to fit the 3 year and 5 year default probabilities implied from CDS quotes (we obtained single-name implied default probabilities using the Bloomberg terminal function CDSW).

Second, we calibrate the model to CDO tranche quotes. Let $\phi_{i}, \phi_{H, s}, \phi_{G, g}$ be the Laplace exponents of $S_{t}^{i}, S_{t}^{s}$ and $S_{t}^{g}$, respectively. Our model implies $\varphi_{i}=\phi_{i}(1)+\sum_{s=1}^{2} \phi_{H, s}\left(H_{i, s}\right)+\sum_{g=1}^{10} \phi_{G, g}\left(G_{i, g}\right)$. Thus, for each $i$, the sum of $\phi_{H, s}\left(H_{i, s}\right), s=1,2$ and the corresponding $\phi_{G, g}\left(G_{i, g}\right)$ must equal some value less than the $\varphi_{i}$ derived in the first step. To determine the value of these Laplace exponents, we first rank 125 obligors in terms of their 5 year default probabilities from low to high, and divide the obligors based on the ranking into two groups. The 72 companies with lower default probabilities are put into the first group (higher credit quality group), and the rest 53 riskier companies to the second group (lower credit quality group). Denote the group index as $r$. For each $r \in\{1,2\}$, we assign three weighting coefficients $w_{H, s}^{r}, s=1,2$ and $w_{G}^{r}$ such that $w_{H, 1}^{r}+w_{H, 2}^{r}+w_{G}^{r}<1$. For each company $i$ in group $r$, we set $\phi_{H, s}\left(H_{i, s}\right)=w_{H, s}^{r} \varphi_{i}$, and $\phi_{G, g}\left(G_{i, g}\right)=w_{G}^{r} \varphi_{i}$. We also note that the parameter $\eta$ in gamma and compound Poisson subordinators can be removed by scaling the weighting coefficients - we only need to determine $C$ for gamma subordinator, and we only need to determine $C / \eta$ for compound Poisson. In total, there are 3 parameters for all the systematic and group subordinators and 6 weighting coefficients to be determined. Thus, the model specification is quite parsimonious.

The following algorithm summarizes the calibration scheme.

\section{Algorithm 2 (Calibration Algorithm)}

1. For each $i$, determine $\varphi_{i}$ to match marginal default distribution based on 3 year and 5 year CDS spreads.

2. Set initial value for $w_{H, 1}^{r}, w_{H, 2}^{r}, w_{G}^{r}, r=1,2$ and $\Omega$.

3. Calculate the Laplace exponents and determine loading coefficients $H_{i, s}$ and $G_{i, g}$. 
Sun, Mendoza-Arriaga, and Linetsky

Table 1: Market quotes and model estimated spreads with simulation standard errors.

\begin{tabular}{ccccc}
\hline \hline Tranche & $0-3 \%$ & $3-7 \%$ & $7-15 \%$ & $7-15 \%$ \\
Market mid(bps) & 3700.0 & 1775.0 & 300.5 & 50.0 \\
Model spreads(bps) & 3701.8 & 1774.9 & 301.8 & 48.3 \\
Market bid(bps) & 3675.0 & 1752.0 & 288.0 & 43.0 \\
Market ask(bps) & 3725.0 & 1798.0 & 313.0 & 57.0 \\
Standard error(bps) & 12.8 & 7.2 & 5.3 & 2.4 \\
\hline
\end{tabular}

Table 2: Calibrated global and group subordinators parameters and weighting coefficients.

\begin{tabular}{cc||cc}
\hline \multicolumn{2}{c||}{ Subordinator Parameters } & \multicolumn{2}{c}{ Weighting Coefficients } \\
\hline$S_{t}^{s=1}$ & $C=2.06 e^{-4}$ & $w_{H, 1}^{1}=0.6012$ & $w_{H, 1}^{2}=0.4626$ \\
$S_{t}^{s=2}$ & $C / \eta=0.0988$ & $w_{H, 2}^{1}=0.2796$ & $w_{H, 2}^{2}=0.1183$ \\
$S_{t}^{g}(\mathrm{~g}=1, \ldots, 10)$ & $C=6.02 e^{-4}$ & $w_{G}^{1}=0.0737$ & $w_{G}^{2}=0.4052$ \\
\hline
\end{tabular}

4. Applying algorithm 1 to calculate $\mathrm{CDO}$ tranche spreads by simulation.

5. Repeat from step 2 to minimize the tranche pricing error. That is, search for $w_{H, 1}^{r}, w_{H, 2}^{r}, w_{G}^{r}, r=1,2$ and $\Omega$ so that the root mean square error of the model produced spreads and market quoted spreads is minimized.

The calibration result is presented in table 1, based on 100,000 replications in the simulation algorithm. The estimated spreads from the model are not only within bid-ask spreads, but within 2 basis points of the middle quotes for all tranches. This illustrates that the dependence structure of the model is rich enough to capture the correlation structure implied in the market quotes for CDO tranches. Calibrated parameters of systematic and group subordinators as well as weight coefficients are presented in table 2 .

\section{CONCLUSION}

The paper develops valuation of multi-name credit derivatives, such as collateralized debt obligations (CDOs), based on a novel multivariate subordinator model of dependent default (failure) times. The default times of individual firms are modeled as first passage times of coordinates of the multidimensional subordinator above independent unit-mean exponentially distributed levels. The model can account for high degree of dependence among defaults of multiple firms in a credit portfolio and, in particular, exhibits positive probabilities of simultaneous defaults of multiple firms. The paper proposes an efficient simulation algorithm for fast and accurate valuation of CDOs with large number of firms and applies it to the calibration of this class of models to CDX market data.

\section{ACKNOWLEDGMENTS}

This research is supported by the NSF grant CMMI-1030486.

\section{REFERENCES}

Barndorff-Nielsen, O.and Pedersen, J., and K. Sato. 2001. "Multivariate Subordination, Self-decomposability and Stability". Advances in Applied Probability 33:160-187.

Barndorff-Nielsen, O. 1998. "Processes of Normal Inverse Gaussian Type". Finance and Stochastics 2:41-68. Bertoin, J. 1996. Lévy Processes. Cambridge University Press. 
Boyarchenko, N.and Levendorski, S. 2002. Non-Gaussian Merton-Black-Scholes Theory. World Scientific Publishing Company.

Cont, R., and A. Minca. 2007. "Reconstructing Portfolio Default Rates from CDO Tranche Spreads". Working Paper.

Cont, R., and P. Tankov. 2004. Financial modeling with jump processes. London: Chapman \& Hall/ CRC.

Das, S., D. Duffie, N. Kapadia, and L. Saita. 2007. "Common Failings: How Corporate Defaults Are Correlated". The Journal of Finance 62:93-118.

Duffie, D., and N. Gârleanu. 2001. "Risk and Valuation of Collateralized Debt Obligations". Financial Analysts Journal 57:41-59.

Duffie, D., and K. Singleton. 1999. "Modeling Term Structure of Defaultable Bonds". Review of Financial Studies 12:687-720.

Embrechts, P. 2009. "Copulas: A Personal View”. The Journal of Risk and Insurance 76:639-650.

Errais, E., K. Giesecke, and L. Goldberg. 2007. "Pricing Credit from the Top Down with Affine Point Processes". Working paper.

Giesecke, K., and B. Kim. 2007, dec. "Estimating Tranche Spreads by Loss Process Simulation". In Proceedings of the 2007 Winter Simulation Conference, edited by S. G. Henderson, B. Biller, M.-H. Hsieh, J. Shortle, J. D. Tew, and R. R. Barton, 967-975. Piscataway, New Jersey: Institute of Electrical and Electronics Engineers, Inc.

Jarrow, R., and S. Turnbull. 1995. "Pricing Options on Financial Securities Subject to Credit Risk". Journal of Finance 50:53-85.

Jorion, P., and G. Zhang. 2007. "Good and Bad Credit Contagion: Evidence from Credit Default Swaps". Journal of Financial Economics 84:860-883.

Kallsen, J.and Tankov, P. 1986. "Characterization of Dependence of Multidimensional Lévy Processes Using Lévy Copulas". Journal of Multivariate Analysis 97:1551-1572.

Lando, D. 1998. "On Cox Processes and Credit Risky Securities”. Review of Derivatices Research 2:99-120.

Li, D. 2008. "On default correlation: a copula function approach". Fixed Income 9:43-54.

Longstaff, F., and A. Rajan. 2008. "An Empirical Analysis of the Pricing of Collateralized Debt Obligation". The Journal of Finance 63:529-563.

Madan, D., P. Carr, and E. Chang. 1998. "The Variance Gamma Process and Option Pricing”. European Finance Review 2:79-105.

Madan, D. B., and E. Seneta. 1990. "The Variance Gamma (V.G.) Model for Share Market Returns". The Journal of Business 63:511-524.

Mai, J.-F., and M. Scherer. 2009. "A Tractable Multivariate Default Model Based on a Stochastic Timechange". International Journal of Theoretical and Applied Finance 12:227-249.

Marshall, A., and I. Olkin. 1967. "A Multivariate Exponential Distribution". Journal of the American Statistical Association 62:30-44.

Mendoza-Arriaga, R., and V. Linetsky. 2010. "Modeling Default Correlation and Clustering: A Multivariate Stochastic Time Change Approach". Working Paper.

Mortensen, A. 2006. "Semi-analytical Valuation of Bastket Credit Derivatives in Intensity-based Models". Journal of Derivatives 13:8-26.

Peng, X., and S. Kou. 2009. "Default Clustering and Valuation of Collateralized Debt Obligations". Working Paper.

Sato, K. 1999. Lévy processes and infinitely divisible distributions. Cambridge University Press.

Schilling, R., R. Song, and Z. Vondracek. 2010. Bernstein Functions. De Gruyter, Berlin.

Schoutens, W. 2003. Lévy Processes in Finance: Pricing Financial Derivatives. John Wiley and Sons, New York.

Sun, Y., R. Mendoza-Arriaga, and V. Linetsky. 2010. "Marshall-Olkin Multivariate Exponential Distributions, Multidimensional Subordinators, Efficient Simulation, and Applications to Credit Risk". Working Paper. 
Zheng, H.and Jiang, L. 2009. "Basket CDS Pricing with Interacting Intensities". Finance and Stochastics 13:445-469.

\section{AUTHOR BIOGRAPHIES}

YUNPENG SUN is a doctoral student in the Department of Industrial Engineering and Management Science at Northwestern University. His research concerns simulation methods and applications in financial engineering. His email address is yunpeng.sun@u.northwestern.edu.

RAFAEL MENDOZA-ARRIAGA is an assistant professor in the Department of Information, Risk and Operations Management at McCombs School of Business at the University of Texas Austin. He completed his Ph.D. in the Department of Industrial Engineering and Management Science at Northwestern University. His current research concerns various aspects of credit risk, including unified credit-equity modeling and modeling portfolio credit risk. His email address is rafael.mendoza-arriaga@ mccombs.utexas.edu.

VADIM LINETSKY is Orrington Lunt Professor in the Department of Industrial Engineering and Management Science at Northwestern University. His research interests are in financial engineering, mathematical and computational finance, and stochastic modeling. His email address is linetsky@iems.northwestern.edu. 